\title{
Agile start-up Business Planning and Lean Implementation Management on Democratic Innovation and Creativity.
}

\author{
Evangelos Markopoulos ${ }^{1}$, Onur Umar ${ }^{1}$, Hannu Vanharanta ${ }^{2}$ \\ ${ }^{1}$ HULT International Business School, Hult House East, 35 Commercial Rd, E1 1LD, London, \\ United Kingdom \\ ${ }^{2}$ University of Vaasa, School of Technology and Innovations, Wolffintie 34, 65200 Vaasa, \\ Finnland \\ evangelos.markopoulos@faculty.hult.edu,onurumar@hotmail.com, hannu@vanharanta.fi
}

\begin{abstract}
Startups are the entities developed by innovative minds aiming to change the world and disrupt the industry. However, those minds are often trapped in what they want to do and on what they have to do. Planning a business based on disruptive visions can contradict the logic that all business must be fast profitable and sustainable. One way to resolve this challenge is by democratizing the business planning process for the knowledge elicitation and commitment confirmation. This paper provides to start-ups, young entrepreneurs and ambitious innovators a practical methodological framework for democratic and agile business plan development, supported with templates and metrics for collective thinking and lean management. Under the applied philosophy for management and leadership concepts and driven by the Delphic maxis of miden-aga, metron-ariston and know-thyself, the framework balances first the nurturing of brilliant minds and then the management and leadership of their brilliant ideas, insights and innovations.
\end{abstract}

Keywords: Business Plan · Company Democracy · Agile $\cdot$ Lean $\cdot$ Management - Applied philosophy $\cdot$ Entrepreneurship $\cdot$ Startup $\cdot$ methodology.

\section{Introduction}

Globalization redefined the way how business established and operates. The last ten years the entrepreneurial roadmap has been changed massively and impressively due to the start-up phenomenon. The opportunities offered through the .com dream, especially to younger generations to reach success with an application or change the world with a smart idea creates a global competition of dreams accomplishment than actual business proposition and development. The gap creates between the desired and the feasible outcome, can be bridged with realistic business planning which combines the innovative creativity needed for the futures state but also the conditions, and restrictions needed to be considered at the present stage. Emphasizing and focusing on what can be deliver 
under specific time, budget and recourses is what differentiates the ones determined to succeed who see seek a shoot to the dream.

However, what determines a well thought business plan remains a fuzzy issue. The completeness of a business plan based on what investors expect or require does not assure the correctness of the information used, projected and forecasted over an operations period. The number or assumptions in a business plan is high and quite accepted from the investors as a plan is a plan, but the degree of realism cannot quite measurable, and this is where the challenge begins.

Today there are many plan methods, templates, tool and practices guiding young entrepreneur, and not only, towards planning their business property. Nearly all of them expect a holistic planning approach with deep understanding of the target market, operations management, marketing, production, sales, financial planning and much more. Such expectations require planning expertise rarely in startups and SME's who seek more practical approached to balance what they want to do today with how they will be in the future or when they can achieve high returns on investment. This would not have been a major issue if the SMEs were not the backbone of every economy [1].

To tackles this issue and support the entrepreneurship movement, business planning shall be approached with methodological frameworks that can adjust the planning process by balancing the creativity and operations, One way to achieve such differentiation is the introduction of democratic, agile and lean business planning frameworks and not actual methodologies. The element of democratization provides the space needed for knowledge to mature in a co-evolutionary way with all involved the project, idea or initiative. Collective thinking can generate effective knowledge which can be transformed into realistic busies plan via agile planning process and later on via lean project implementation management.

\section{The Startup Entrepreneurial Revolution.}

The evolution of technology, the computing power, the rise of the internet, the computing mobility, accessibility, user friendliness, the global connectivity, the digital services and many other factors which got aligned at the same time with the same pace created the circumstances for a new business revolution. The startup revolution is led primarily by the millennials, or the liberal Y-generation, which embraces the concept as an opportunity to crave more meaning in their lives through their vision to change the world or live their myth. They find it easier than ever to start a business and an opportunity to take control over their lives, as they do not trust their leaders. They feel that they do not have anything better to do and nothing to lose. They are not afraid to fail knowing that most of the startups fail. Today, $99 \%$ of the startups who request funding are rejected, and nearly $75 \%$ of those who receive venture capital funding never return their investment [2]. Therefore, they can safely go after their dream without counting a failure as a failure.

The entrepreneurial movement which also grows tremendously in the universities worldwide has supported this trend. New academic programs with emphasis on entrepreneurship of any kind teach millions of students each year how to utilize their skills through the opportunities startup offer. Today nearly 133 million startups are created annually [3]. This is 4 startups per second, with only $0.05 \%$ of them to reach basic 
funding. More than half of the funded ones, nearly 50.000 are in the US, and about 1.500 of them reach VC funding which is 1 out of 88.667 or $0.00112 \%$.

The United States leads this startup revolution with tremendous amounts of investments each year. During the period of 2011-2014 nearly \$140 billion were invested by venture capitalists on nearly 12 thousand deals. This is an average of $\$ 35$ billion per year and an average of $\$ 12$ million per deal [4]. The technology sector (Internet, mobile, software, electronics \& hardware) absorbing in $2014,75 \%$ of all VC deals, making an increase of $73 \%$ from 2013 . However it is not all the US that spends such amounts on startups. The top 5 states with the most deals in 2014 were California (1.631), New York (422), Massachusetts (346), Texas (143) and Washington (97), while 23 states, nearly half the country, had under ten deals each.

\section{Business Plan methods, model and frameworks.}

The first step on the startup entrepreneurial journey is the creation of a business plan through which the overall idea is mapped on it, to be evaluated for its technical, financial and investment feasibility. To cope with this revolution, Business Plan templates have been developed from various organizations, institutions and private corporations, with different business planning approaches and similar steps, differentiated in the order of execution and on the depth of the requested documentation. The Business Model Canvas, the Lean Canvas, the McKinsey Template, the HSBC Template and the IBM Watson Build Business Plan Template are briefly presented and analyzed.

The Business Model Canvas (BMC) is a chart-based template that visually documents nine key areas of new or existing business. The canvas collects information for each area and provides an organizational blueprint based on which further planning and action can take place. The BMC areas are the Key Partners, Key Activities, Key Recourses Value Proposition, Channels, Customer Segment, Cost Structure, and Revenue Streams [5], [6]

The Lean Canvas is a simpler version of the Business Model Canvas with emphasis mostly on the idea than the business operations. The lean canvas maintains the nine areas of the Business Model Canvas but replaces the 'Key Partners' with the 'Problem', the 'Key Activities' with the 'Solution', the 'Key Recourses' with the 'Key Metrics' and the 'Customer Relationship' with the 'Unfair Advantage'. The Problem-SolutionMetrics-Advantage structure reduces the operations complexity of the BMC and makes business planning more practical and simplified. [7]

The McKinsey Business Plan structure is related with the McKinsey three horizons of innovation. The planning model has specific elements that get completed in depth and gradually over the maturity of the innovation. This completion takes place in three stages where in the first stage key elements and individual topics are addressed were at the last stage the integration of all the elements form the total business plan. The elements are the Product Idea, Competencies and Profiles of the management team, Product Marketing possibilities, Company Operations, Detailed Schedule of the company realization, Financial Planning and the Risks Involved [8].

The HSBC Business Plan template has a more financial approach. The template includes a Brief Business Description, a Business Overview, Markets and Competition, Sales and Marketing, Management, Operations, Forecasts and Financing. As the model 
derives from the financial sector it emphasizes more on the tangible and objectives financial elements than the subjective ones related to the innovation, market disruption and potential growth [9].

The IBM Watson Build Business Plan Template is a tool under the IBM's Watson Build global initiative that has been designed to accelerate the adoption of Watson by the IBM Business Partners' and encourage the development of AI solutions on the IBM Cloud. The main elements of the business plan are the Big Idea, Solution, Target Market, Market Need, Competition, Team, Budget and Sales Goals, Sales and Marketing, Risk Assessment, and Milestones [10].

\section{The dilemma between Planning the Business and Business Planning}

Startups and entrepreneurs are usually in the dilemma to plan their business based on the vision or on the profit. Planning a business to disrupt the market and attract visionary investors, does not necessarily has to be profitable in the early periods of its operations. However, planning a business that can create fast and quick profit, which can attract finance-oriented investors, need a solid financial plan regardless the degree of innovation or disruptiveness. Therefore, the dilemma entrepreneurs have is either to plan their business on do business planning. The first one is based on the vision and disruptiveness while the second is based on the financial sustainability and profitability.

Shining startups are the ones who can see the innovation, the vision, the disruption. There are the ones who create blue oceans and generate new eras in the economy, the markets and the society impacting the end users, the buyers, and the consumers.

Prior emphasizing on how fast a startup will generate profit, return on investment, and wealth, it is more important to emphasize on innovation itself [11]. It is the 'wow' that shall proceed the 'how'. There have been numerous success stories of startups that made global impact with very poor initial financial plans [12], and others that operated in loss for many years, prior reaching profitability and success [13].

Business plans and financial plans above all are pure estimations based on research under hypothesis rarely able to be proved sufficiently. This, however, makes sense as startups being in early stages cannot provide solid business plans, with actuall financial data from business operations that can be used to develop future estimations through which investments will be attracted.

Startups have the charisma to think out of the box and of course out of the numbers. The pressure to justify every business operation with financial data restricts innovative thinking, vision and ideas. Start-uppers have the dream, and this is where innovators shall emphasize in the initial stage. Today there is surplus of funds to be invested but there is a scarcity of ideas with meaningful impact to attract investors who first want to be change makers and then to obtain profits from their success. According to Forbes, the eight factors investors are seeking to see in a startup are all related with the vision and the opportunity [14]. Specifically, they look for the person as an entrepreneur and the overall team, the mission, the size of the opportunity, the quality of the presentation, presentation, the value investors can add, the timing of the market, the research conducted and if anyone else is investing. 
An alternative evaluation of a startup idea valuation has to do with the degree of uncertainly the idea has. This degree can be calculated by various economic, political, social, technological, legal, and other factors. The degree of acceptance an idea has, can affect heavily investment decisions. Also, the maturity (readiness) of the idea has to be safely and widely adopted in the market, in the legal framework that can support such operations or in the political environment that can create the stability in the target country the startup intends to operate initially, impacts as well the investment decision-making process [15]. The degree of such considerations can be calculated with probabilities, but again this remains a subjective approach to accurately identify the uncertainty of the business plan and reject it as insufficient. High uncertainty does not necessarily mean high investment risk. On the contrary, high uncertainly can mean low investment risk as the profit loss from not investing on something that not quite clear and miss the opportunity to disrupt the industry, can be tremendous.

In both approaches, the vision-based oriented one, and the uncertainty-based one, the financial analysis pays the least significant role. According to data, cases and experiences, a strong vision and market, makes the financial estimations less important [14]. Therefore, the dilemma remains between planning the business (vision driven) and business planning (finance driven). The entrepreneur shall decide vision discounts to be more affordable and fast profitable or insist on the vision hoping to find mind-like investors.

\section{Democratizing the Business Planning process.}

One approach to overcome the planning the business or business planning dilemma is to democratize the innovative thinking in the sense that a co-evolutionary business planning can be achieved with the contributions of all, starting from the vision all the way to the well thought financial estimations or operations. This collaborative thinking is a continuous process or knowledge sharing and experimentation towards the optimizations of a business idea.

Such a democratization can be achieved through the Company Democracy Model (CDM). The Company Democracy Model [16], [17] uses the wisdom of the ancient Hellenic Delphic maxims [18], with emphasis on the 'Gnothi Seauton', 'Metron Ariston'and 'Miden Agan'. The model operates in a spiral evolutionary process through which a knowledge-based democratic culture aligns personal and organizational goals. This co-evolutionary approach identifies the achievements, the capacity, capability, competence, and maturity of the human resources, and turns such data, information, and knowledge into innovations. The model is aligned with the Evolute methodology which optimizes knowledge management for continuous innovation development [19].

The CDM can be adjusted to contribute on resolving this startup dilemma. Figure 1 presents the six (6) levels of the adjusted model and the activities that take place in each. The six levels of the startup CDM are divided into two groups of activities that represent the vision planning and the financial planning approach.

Levels 1-3 emphasize on the creative and innovative part of the business plan. This is achieved initially on Level 1, with the optimization of the feasibility of the idea in 
terms of innovation and disruptiveness through continuous democratic knowledge elicitation from all who can contribute. Level 2 emphasizes on team building and management for maximum performance optimization and the development of working prototypes. Level 3 aims on extending the development of the working prototypes into operational prototypes, as well as product/service validation and verification that will clarify the realization of the innovation and contribute to its capitalization.

Levels 4-6 can contribute of the business operations dimension. Level 4 is based on the innovation effectiveness in order to identify the markets to operate best and based on that to analyze the operations costs and expected revenues. Having identified and tested both the innovation and the market, level 5 creates the competitive advantages and the communication strategy that will be disseminated by marketing initiatives to attach clients and partners. Lastly, level 6 identifies the breakeven point for further investors, global operations, IPO, strategic partnerships, etc., or the exit or de-investment strategy if that is more suitable per case.

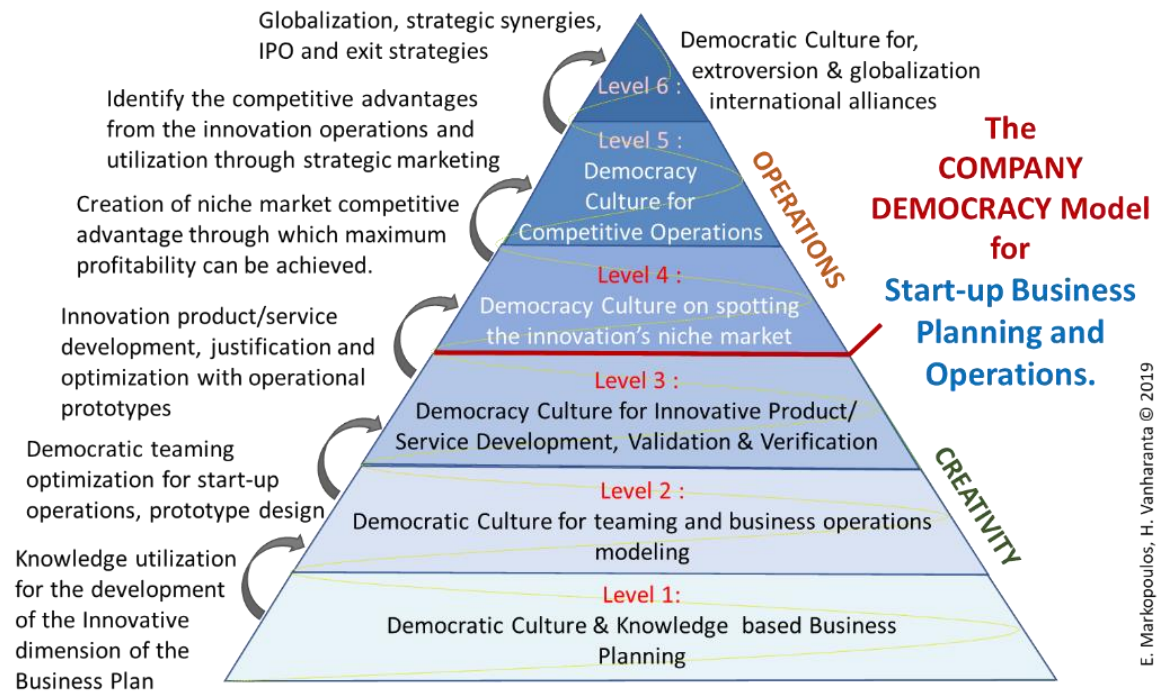

Fig. 1. The adjusted Company Democracy Model for the democratization of startup business planning and operations

\section{The Democratic Agile Start-Up Business Plan (DASBP)}

The Democratic and Agile Business Plan (DASBP) can be a new business-planning model to address the startup dilemma. The model approaches the planning process first for the creativity dimension and then from the financial. The creativity approach is aligned with the idea that the startups must emphasize first on the innovation that will support the business. Lack of innovation in products or services cannot assure long and or fast success and sustainability. Business plans with weak innovative products or services can be mostly considered theoretical or ambitious. Having defined, developed and tested the innovation, business planning becomes more realistic and predictable. 
This balance between the innovative and operations dimension of a business plan is characterized by the agility offered to the innovator / entrepreneur to develop first the idea and then define the business based on the degree of innovation. This impacts the degree of competitiveness and helps safe operations predictions to be made. The two dimensions can be view as two layers of the business planning process. The first layer of the DASBP has six key elements that must be completed prior moving to the second layer. They are: Value, Growth, Transparency, People, Market and Agility (Fig. 2).

The value of the startup indicates the uniqueness or the idea, the degree of the innovation, and the benefit to the clients and the market. Growth indicates potential growth or the sectors in terms of innovation disruptiveness. The market indicates the existing total available market segmentation. The team indicates capability, maturity and capacity of the start-up human recourses and teaming structure. The transparency is related with the clarity on the innovation in terms that innovation is well defined and communicated. The agility is related with the degree of flexibility the innovation has when it needs to be changed or adjusted to various circumstances

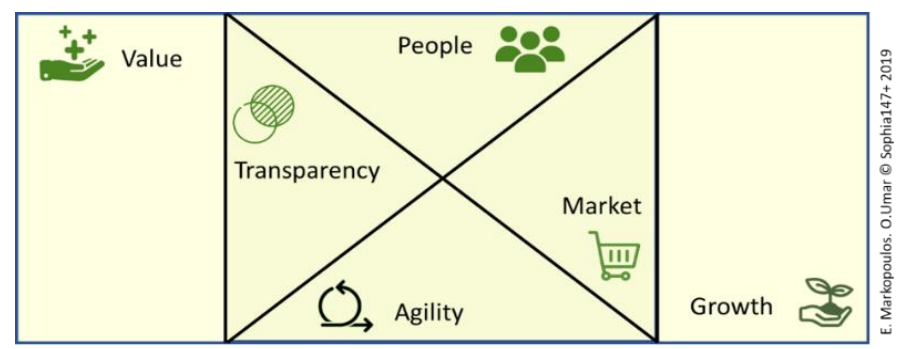

Fig. 2. The first, innovative, layer of DASBP

The second layer of the DASBP has also six key elements, which are the partners, revenue, suppliers, price, client relation, and operations cost (Fig. 3.). Partners indicate the strategic partnerships made or need to be made for the start-up success. Revenue presents the revenue expected streams. Suppliers indicate the material and operations support needed. Price indicates the pricing scheme. Client indicates the relations communication, dissemination, marketing and client support. Operational costs indicate the fixed, contingency and risk management costs.

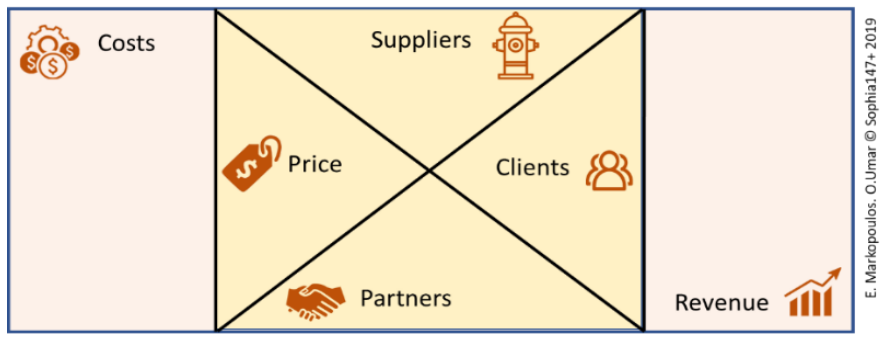

Fig. 4. The second, operations, layer of DASBP 
The integration of the two layers form the holistic version of the DASBP model. The model is read from bottom to top going through the Startup CDM version evolution of knowledge maturity to innovation with the proper team structure and a series of prototypes (levels1-3) that verify innovation existence and acceptance, prior the identification of the market segments, the competitiveness and the global sales strategy (fig. 4). The distance between layers one and layer two is related with the time needed for the maturity, development and validation of the innovation.

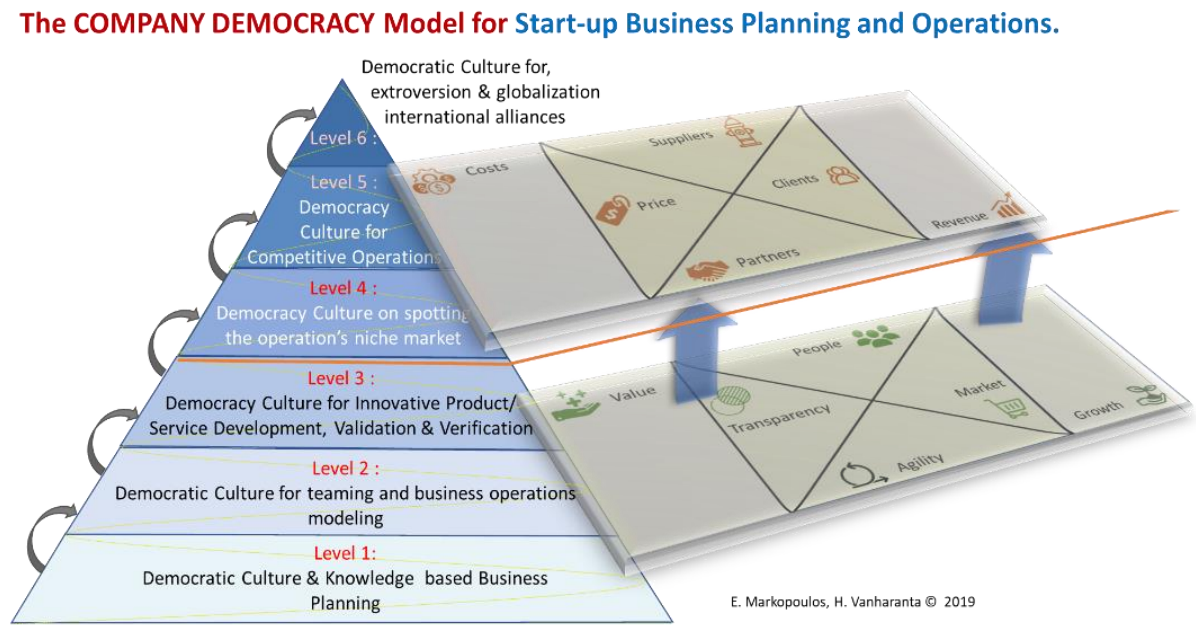

Fig. 5. The DASBM models in the CDM framework.

\section{DASBP application Metrics and indexes.}

The effectiveness of a business plan can either be illusionary or have a subjective value based on the one who creates it or judges it. The Business Model Canvas, which is today a widely used framework does not record any data and does not provide any value indicators that could be used to score the effectiveness or the overall value of the business plan. No attempt has been indicated so far to quantify the effectiveness of a business plan, however the agility and staged development offered through the DASBP minimizes the business plan elements, groups them, prioritizes them, and makes the development of metrics feasible.

Based on this, a three dimensional DASBP metric model can be developed to deal with the importance of the elements of each layer, the correctness and the degree of completion. This scoring can be achieved by initially providing a weight to each element. This weight can be related with the potential of the industry which can be found in the Bloomberg, S\&P500, and other ratings. The total weight of the elements based on the industry value can provide the overall weight, in terms of the business plan value.

Furthermore, the degree of complements can be calculated by counting the number of requirements each layer's element requires to be completed. As of today, no business plan provides a minimum set of required elements in each planning category. This creates a great degree of fuzziness in the correct execution of the business planning 
process. The last metric can be associated with the correctness of the completion. This can be justified with documentation provided for the fulfillments of each requirement of each element of a DASBP layer. The documentation needed can also be guided by a set of minimum references that justify the qualitative completion of an element requirement (Fig. 5).



Fig. 5. Computation elements structure in the DASBP weighing system.

It must be noted that incomplete element requirements impact the business plan weight. In the same sense, completion requirements, or corrections requirements not completed, impact as well the business plan score. Equation 1 presents the weight calculation process (1). The complete total business plan weight has all its elements weighted with the maximum values. However, and as this an agile and staged approach the weight can be divided in the two layers and investments can be made based on the planning progress through the completion of each DASBP layer.

Business Plan Weight $=$ Industry Value $*$ Total Elements Requirements Weight * (Total Completeness * Total Correctness) .

The DASBP weight approach is a theoretical start to business plans validation and a contribution towards standardizing the startup business planning process which distributes today tremendous investments through subjective or bureaucratic judgement.

\section{Conclusions}

The challenge of business planning or planning the business has been and will remain a major concern on investment management and market evaluation. Regardless the stage of an organization, in terms of operations, the business plan either it is for a startup or for the realization of global strategies in an established organization, contains to a great degree a number of estimations that impact significantly the outcome of its execution. Staged, agile and weighted business planning can reduce such risks, to an extent, due to the distribution of the planning commitments in stages and the investment 
commitments on the correctness of each stage. The DALBP model attempts to contribute on this challenge as an initial approach by introducing three innovations. First is the democratization of the knowledge in thinking and planning, second is the staged planning from the innovation to the operations and third is the weighting calculation to size the volume of the effort. DALBP links of the previous two contributions with the investment decision making and the degree of investment that can be made per case. The model is at an initial stage and further research and development will follow, as well as industry applications towards the optimization of the layer's elements, completion requirements, correction requirements, and metrics computations.

\section{References}

1. OECD Observer. http://www.oecd.org/cfe/leed/1918307.pdf

2. Forbes. https://www.forbes.com/sites/85broads/2013/11/18/the-millennial-startup-revolution/\#536ecfa9622d

3. Global Entrepreneurship Monitor https://www.gemconsortium.org/report

4. The 2014 US Venture Capital Report. Year in Review. Financial and Exit analysis. CP Insights. (2015)

5. Oyedele A: Emerging market global business model innovation; Journal of Research in Marketing and Entrepreneurship, vol. 18 Issue: 1, pp.53--62 (2016)

6. Osterwalder A., Pigneur Y.: Business Model Generation: A Handbook for Visionaries, Game Changers, and Challengers Paperback. NJ. USA (2010).

7. Arwa A., Jamaludin I.: Business Model Canvas, the Lean Canvas and the Strategy Sketch: Comparison' International Journal of Scientific \& Engineering Research, vol 9, issue 1, January-2018 (2018)

8. Kubr T., Marchesl H., Ilar D.: Starting-Up. Achieving success with professional business planning. McKinsey \& Company, Netherlands (1998)

9. HSBC. https://www.knowledge.hsbc.co.uk/business_plan/embed

10. IBM. https://www-356.ibm.com/partnerworld/wps/servlet/ContentHandler/watson-build

11. Harvard Business Review. https://hbr.org/2012/05/great-businesses-dont-start-wi

12. Forbes. https://www.forbes.com/sites/kevinkruse/2018/07/30/the-no-plan-business-plan-forentrepreneurs/

13. Entrepreneur Europe. https://www.entrepreneur.com/article/272710

14. Forbes. https://www.forbes.com/sites/alejandrocremades/2019/01/09/how-investors-decideto-invest-in-startups/

15. Jean-Charles Pomerol J-C.: Business uncertainty, corporate decision and startups, Journal of Decision Systems, 27: sup1, pp 32--37 (2018)

16. Markopoulos E., Vanharanta H.: Democratic Culture Paradigm for Organizational Management and Leadership Strategies - The Company Democracy Model. Proceedings of the 5th International Conference on Applied Human Factors and Ergonomics (2014).

17. Vanharanta H., Markopoulos E.: Creating a Dynamic Democratic Company Culture for Leadership, Innovation, and Competitiveness. 3rd Hellenic-Russian Forum. Sept.17. (2013).

18. Parke H., Wormell D.: The Delphic Oracle, Basil Blackwell, vol. 1, pp 389 (1956).

19. Kantola, J., Vanharanta, H., Karwowski, W.: The Evolute System: A Co-Evolutionary Human Resource Development Methodology. In the International Encyclopedia of Ergonomics and Human Factors. Karwowski, W., CRC Press. (2006). 